nephron

Practice
Nephron 2018;140:116-119

DOI: $10.1159 / 000492064$
Received: June 29, 2018

Accepted after revision: July 12, 2018 Published online: August 2, 2018

\title{
Acute Kidney Injury in Real Time: Prediction, Alerts, and Clinical Decision Support
}

\author{
F. Perry Wilson ${ }^{a, b}$ Jason H. Greenberg ${ }^{a, c}$ \\ a Program of Applied Translational Research, Yale University School of Medicine, New Haven, CT, USA; ${ }^{b}$ Department \\ of Internal Medicine, Section of Nephrology, Yale University School of Medicine, New Haven, CT, USA; ${ }^{\mathrm{C}}$ Department \\ of Pediatrics, Section of Nephrology, Yale University School of Medicine, New Haven, CT, USA
}

\section{Keywords}

Acute renal injury $\cdot$ Alert $\cdot$ Clinical decision support $\cdot$ Real time modeling

\begin{abstract}
Broad adoption of electronic health record (EHR) systems has facilitated acute kidney injury (AKI) research in 2 ways. First, the detection of AKI based on changes in serum creatinine has largely replaced the sensitive but nonspecific administrative coding of AKI that predominated in earlier studies. Second, the ability to implement real-time AKI interventions such as alerts and best-practice advisories has emerged as a promising tool to fight against the harmful sequela of AKI, which include short-term adverse outcomes as well as progression to chronic kidney disease, dialysis, and death. In

Contribution from the AKI and CRRT 2018 Symposium at the 23rd International Conference on Advances in Critical Care Nephrology, Manchester Grand Hyatt, San Diego, CA, USA, February 26 March 1, 2018. This symposium was supported in part by the NIDDK funded University of Alabama at Birmingham-University of California San Diego O'Brien Center for Acute Kidney Injury Research (P30DK079337).
\end{abstract}

\section{KARGER}

() 2018 S. Karger AG, Basel

E-Mail karger@karger.com

www.karger.com/nef this review, we discuss the current state-of-the-art in EHRbased tools to predict imminent AKI, alert to the presence of $\mathrm{AKI}$, and modify provider behaviors in the presence of AKI.

(c) 2018 S. Karger AG, Basel

Acute Kidney Injury (AKI) affects between 10 and $15 \%$ of hospitalized patients and is associated with a nearly 10 -fold increased risk of inpatient mortality [1]. AKI is also associated with adverse long-term outcomes such as hypertension, chronic kidney disease, end-stage renal disease, and mortality [2]. In response to an increasing rate of AKI among hospitalized patients, coupled with concerns that AKI may be in some cases iatrogenic or improperly managed, the Centers for Medicare and Medicaid Services have proposed that AKI be added to a monitored list of inpatient harms that can be used to determine hospital reimbursement rates [3]. In this environment, predicting AKI before it occurs and responding appropriately to AKI when it develops is an issue of high importance to patients, physicians, and healthcare systems alike. Still, we are only beginning to see the implementation of real-time interventions that hold the promise to change patient care and outcomes.

\footnotetext{
Dr. F. Perry Wilson

Department of Internal Medicine, Section of Nephrology

Yale University School of Medicine

60 Temple Street, 6th Floor, Suite 6C, New Haven, CT 06510 (USA)

E-Mail francis.p.wilson@yale.edu
} 


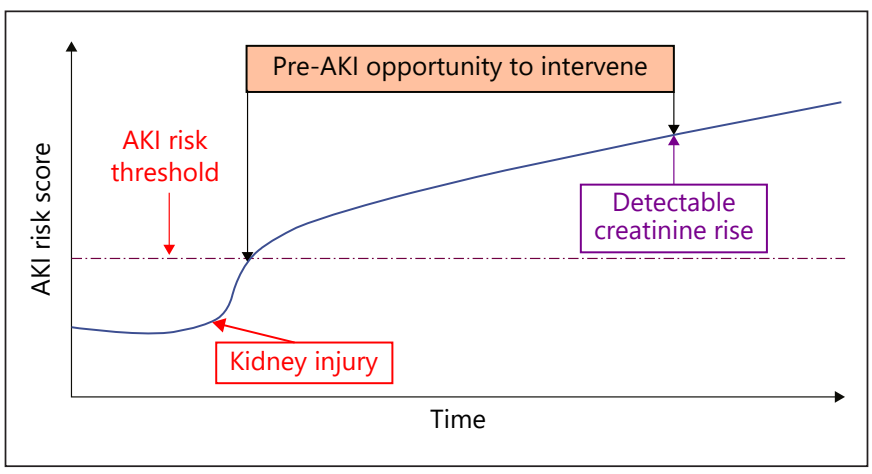

Fig. 1. A clinical model using time-updated covariates is used to generate a dynamic acute kidney injury (AKI) risk score, which reflects the probability of the development of AKI within a given time period (e.g., 24 or $48 \mathrm{~h}$ ). Beyond a predetermined threshold of risk, alerts are triggered to allow targeting of diagnostic and therapeutic strategies prior to the patient meeting the definition of AKI by creatinine criteria.

\section{Prediction of AKI}

Disappointing results from several trials aimed at ameliorating AKI once it has developed has led to significant interest in predicting the onset of AKI before the creatinine starts to rise. The bulk of the research in this area has been surrounding serum and urine biomarkers, but these tests can be expensive and difficult to access in a clinical setting [4]. Leveraging existing electronic health record (EHR) data to predict imminent AKI is potentially cost-free and may offer similar performance to current biomarker-based prediction. Additionally, EHR-based models may identify patients in whom specific biomarkers should be measured, creating synergy between the 2 predictive modalities.

Creatinine is a later marker of AKI, typically rising between 1 and 3 days after a discrete kidney insult. By the time creatinine rises, significant kidney damage may have already occurred, making it imperative to identify patients at risk of AKI and modify their care as early as possible. This implies a substantial window of opportunity for intervention between the insult and clinical AKI definition (Fig. 1). Most studies that have attempted to predict AKI using clinical variables have relied on static features (such as demographics or comorbidities) or dynamic features (like lab values) measured at a static time point (e.g., ICU admission or post-operatively) $[5,6]$.

The first study to comprehensively use time-varying data to predict AKI was published by Koyner et al. [7]. Their predictive model used time-updated vital signs and lab values in addition to demographic data to predict whether hospitalized patients would develop AKI within the next 24-h, with the risk continuously updating as data was collected during the hospital stay. The model had good discrimination for imminent AKI, with an area under the receiver-operator characteristic curve of 0.74 for prediction of stage $1 \mathrm{AKI}$ and 0.83 for prediction of stage 3 AKI, outperforming static models. The next logical step is to implement these models into the EHR to allow realtime interventions for patients at high or increasing risk of AKI. To date, no such studies have been published.

\section{AKI Alerting}

As AKI can be defined simply by the change in creatinine over time, AKI alerts represent relatively low-hanging fruit for real-time, algorithm-based management of patients. Given that several studies that suggest that AKI often goes unrecognized, and that management decisions may suffer in the absence of recognition, it is no wonder that the number of institutions using AKI alerts has risen dramatically [8]. This effect is seen in the National Health Service in the United Kingdom, where AKI alerting is a universal practice.

Despite the ubiquity of AKI alerts, the data regarding their clinical efficacy is mixed. In a seminal before/after study by Colpaert et al. [9], the provision of AKI alerts dramatically increased the number of patients who received therapeutic interventions for AKI within 1 hour of the alert and demonstrated improvements in AKI stage (though not in other clinical outcomes such as receipt of dialysis, length of stay, and inpatient mortality). Another before/after study by Park et al. [10] evaluated whether an AKI alert system, which automated a targeted nephrology consult for all patients with stage 1 AKI would improve outcomes. While the study showed a $25 \%$ decrease in severe AKI rates and a $70 \%$ increase in renal recovery rates, no mortality benefit was seen. Considering that 1,232 individuals received nephrology consults in this study over a 1-year period, broader adoption of this approach would likely strain many nephrology services.

Another before/after study by Selby et al. [11] examined the effect of alerts coupled with hospital-wide AKI guidelines, an educational program, and an AKI care bundle in 8,411 individuals. This care paradigm was associated with significant improvements in overall survival - making this one of the few studies that found AKI alerts to be associated with improvements in a hard outcome. This study highlights a central principle in the science of implementing electronic alerts: the more "active" components in an alert, the more likely they are to be beneficial. But high-quality alert programs with large educational components are not easily generalized; few hospitals may be able to support them. One approach that 
may increase the effectiveness of EHR alerts is to only target patients expected to benefit from an alert. We demonstrated that older individuals, especially women, with lower serum creatinine levels were more likely to benefit from AKI alerts [12].

To date, only 1 randomized trial has evaluated AKI alerting. The trial in 2,393 patients, conducted by our group, was performed in a single center and sent only a single alert to a single physician (typically the intern caring for the patient) [13]. There was no difference in the primary composite outcome of progression of AKI, dialysis, or death. Currently a large, multicenter randomized trial of AKI alerts is underway that addresses limitations in the aforementioned trial by increasing the sample size to 6,030 patients, enrolling across 6 centers, and delivering alerts to all providers when the EHR of the patient is accessed (clinicaltrials.gov NCT0275375).

\section{Clinical Decision Support in AKI}

Automating clinical decision making in AKI may prove a fertile middle ground between purely informative alerting and full-fledged renal consultation. To date, studies in this vein have focused almost exclusively on mitigating drug toxicity among patients with AKI. In 2010, McCoy et al. [14] published a study that targeted AKI alerts to patients receiving at least one of 122 potential nephrotoxins. The study attempted several different versions of alerting - the most intrusive of which increased the rate of dose modification from 35 to $53 \%, p<$ 0.001 . However, there was no signal of clinical benefit. A similar study in a geriatric population found that drugspecific alerts (coupled with physician education) led to nephrotoxic medication cessation in $62 \%$ of patients compared to a pre-intervention rate of $38 \%$ of patients [15].

Providing actionable clinical decision support before the development of AKI potentially holds the most promise to meaningfully improve AKI outcomes. The most robust implementation of this approach was developed by Goldstein et al. [16] at Cincinnati Children's Hospital. The program identifies children at risk of AKI based on their receipt of nephrotoxic medications and directs their providers to monitor creatinine and potentially to change nephrotoxic medication exposure. A recent analysis of the effect of the program revealed that nephrotoxic medication exposure was reduced by $38 \%$ and AKI rates were reduced by $64 \%$, suggesting that prompt removal of nephrotoxins may provide significant benefit at least in the short term.

It bears consideration what other metrics, aside from drug redosing or cessation, would merit "best practice" designation in the setting of AKI. These best practices may be distinct from AKI quality measures such as readmission rate, length of stay, mortality, or AKI-specific follow-up [17]. The Kidney Disease Improving Global Outcomes international consensus guidelines settle on several criteria that may be considered "best practices" in AKI. These include monitoring of serum creatinine and urine output, avoiding diuretics and radiocontrast, discontinuing nephrotoxic medications, appropriate diagnostic workup (tests such as urinalysis, urine microscopy, and kidney ultrasound), and hemodynamic management [18]. These metrics could be specifically endorsed in the setting of an AKI alert. In addition, "intelligent" alerts that take into account dynamic patient factors may be able to provide specific recommendations along these lines. It is of little use to encourage "hemodynamic support" for patients with normal blood pressures, for example, but might be highly relevant to a patient with hypotension. Finally, EHR-based clinical support can help guide appropriate outpatient follow-up and monitor for a long-term renal recovery after AKI.

In conclusion, there are multiple points in the care of patients at risk of or greatly affected by AKI where realtime EHR-driven interventions may be appropriate. The science of real-time clinical decision support for AKI is still in its infancy, with current research suggesting that while process measures may be easily changed through these efforts, hard clinical outcomes may be more difficult to improve. Nevertheless, as the sophistication of real-time algorithms increase and the "intelligence" of clinical decision support improves commensurately, it is plausible that these tools will take on a major role in our AKI armamentarium.

\section{Disclosure Statement}

The authors have no conflicts of interest to declare.

References

1 Hoste EA, Bagshaw SM, Bellomo R, et al: Epidemiology of acute kidney injury in critically ill patients: the multinational AKI-EPI study. Intensive Care Med 2015;41:1411-1423.

2 Coca SG, Singanamala S, Parikh CR: Chronic kidney disease after acute kidney injury: a systematic review and meta-analysis. Kidney Int 2012;81:442-448.

3 Hsu RK, McCulloch CE, Dudley RA, Lo LJ, Hsu CY: Temporal changes in incidence of dialysis-requiring AKI. J Am Soc Nephrol 2013;24:37-42. 
4 Parikh CR, Coca SG, Thiessen-Philbrook H, et al: Postoperative biomarkers predict acute kidney injury and poor outcomes after adult cardiac surgery. J Am Soc Nephrol 2011;22: 1748-1757.

5 Malhotra R, Kashani KB, Macedo E, et al: A risk prediction score for acute kidney injury in the intensive care unit. Nephrol Dial Transplant 2017;32:814-822.

6 Basu RK, Zappitelli M, Brunner L, et al: Derivation and validation of the renal angina index to improve the prediction of acute kidney injury in critically ill children. Kidney Int 2014;85:659-667.

7 Koyner JL, Adhikari R, Edelson DP, Churpek MM: Development of a multicenter wardbased AKI prediction model. Clin J Am Soc Nephrol 2016;11:1935-1943.

8 Grams ME, Waikar SS, MacMahon B, Whelton S, Ballew SH, Coresh J: Performance and limitations of administrative data in the identification of AKI. Clin J Am Soc Nephrol 2014; 9:682-689.
9 Colpaert K, Hoste EA, Steurbaut K, et al: Impact of real-time electronic alerting of acute kidney injury on therapeutic intervention and progression of RIFLE class. Crit Care Med 2012;40:1164-1170.

10 Park S, Baek SH, Ahn S, et al: Impact of electronic acute kidney injury (AKI) alerts with automated nephrologist consultation on detection and severity of AKI: a quality improvement study. Am J Kidney Dis 2018;71: 9-19.

11 Selby NM: Electronic alerts for acute kidney injury. Curr Opin Nephrol Hypertens 2013; 22:637-642.

12 Biswas A, Parikh CR, Feldman HI, et al: Identification of patients expected to benefit from electronic alerts for acute kidney injury. Clin J Am Soc Nephrol 2018;13:842-849.

13 Wilson FP, Shashaty M, Testani J, et al: Automated, electronic alerts for acute kidney injury: a single-blind, parallel-group, randomised controlled trial. Lancet 2015;385: 1966-1974.
14 McCoy AB, Waitman LR, Gadd CS, et al: A computerized provider order entry intervention for medication safety during acute kidney injury: a quality improvement report. Am J Kidney Dis 2010;56:832-841.

15 Roberts GW, Farmer CJ, Cheney PC, et al: Clinical decision support implemented with academic detailing improves prescribing of key renally cleared drugs in the hospital setting. J Am Med Inform Assoc 2010;17:308-312.

16 Goldstein SL, Kirkendall E, Nguyen H, et al: Electronic health record identification of nephrotoxin exposure and associated acute kidney injury. Pediatrics 2013;132:e756e767.

17 Mehboob A, Zimmerman R, Abramson S, Parker MG: Quality measures in acute kidney injury. Curr Opin Nephrol Hypertens 2018; 27:130-135.

18 Kidney Disease: Improving global outcomes (KDIGO) acute kidney injury work group. KDIGO clinical practice guideline for acute kidney injury. Kidney Int 2012;2(suppl):1-138. 